\title{
versants
}

\section{Dictionnaires et régimes de réputation autour de 1750}

\author{
Jean-Luc CHAPPEY \\ Université Paris I Panthéon-Sorbonne
}

\begin{abstract}
Le dictionnaire historique est un genre littéraire à part entière, inauguré par le Grand dictionnaire historique de Louis Moréri, paru pour la première fois en I674. Largement marqué par les origines religieuses de son auteur, le dictionnaire s'émancipe pourtant, au fil de ses rééditions successives, de sa composante biblique, pour offrir une place de plus en plus importante aux hommes de mérite, et notamment aux Hommes de lettres. Bientôt concurrencé par des projets parallèles - ceux de Bayle au XVII et de Ladvocat au XVIII entre autres - le dictionnaire s'impose progressivement comme un lieu de publication d'une réputation qui dépend du jugement de l'auteur, devenant ainsi un espace de critique littéraire. Pour des raisons historiques, tout autant que sociales ou pratiques, le dictionnaire historique n'échappe pourtant pas au champ des querelles religieuses : à la guerre entre philosophes et clergé s'ajoute alors également la question du conflit entre jansénistes et jésuites.
\end{abstract}

Keywords: dictionnaires, biographie, réputation.

Les « origines » française ${ }^{I}$ du genre du dictionnaire historique sont traditionnellement renvoyées à I674, date de la publication à Lyon du premier volume in-folio du désormais fameux Grand dictionnaire historique ou mélange curieux de l'histoire sacrée et profane de Louis Moréri (I643-I680), aumônier puis prêtre à Lyon qui décède avant d'avoir achevé son travail ${ }^{2}$. Un deuxième volume posthume est publié en I68I grâce au travail de Parayre, premier commis du ministre et secrétaire d'État Pomponne dont les enfants avaient pour précepteur Moréri. L'ouvrage, écrit en français, connaît un succès immédiat comme en témoignent ses rééditions successives à Lyon (I68I, I683,

\footnotetext{
I On sait combien le terme d'origines prête à discussion. L'entreprise de Moréri est précédée de nombreux dictionnaires comme le Dictionnaire théologique, historique, poétique et chronologique de Daniel Juigné Broissière publié en I643. Néanmoins, le Grand dictionnaire est le premier ouvrage de ce type à être rédigé en français.

2 Après avoir suivi ses humanités à Draguignan puis sa rhétorique chez les jésuites d'Aix-enProvence, il est ordonné prêtre à Lyon. Son Grand Dictionnaire ou mélange curieux de l'histoire sacrée et profane est édité à Lyon en I674 en un volume. L'ouvrage est dédié à l'évêque d'Apt, Gaillard de Longjumeau, Moréri étant alors aumônier dans ce diocèse.
} 
I687, I688, I69I³), en Hollande (I696, I698, I7024), puis à Paris (I699, I704, I707), la publication de suppléments dès $1689^{5}$ et ses traductions dans divers États européens. Entre I679 et I759, près de dix-sept éditions sont ainsi publiées, le Dictionnaire passant de deux volumes en I68I à cinq en I7I8 puis à dix volumes in-folio en I749. Première « encyclopédie » alphabétique de " mots et de choses » selon Michel Foucault, le dictionnaire de Moréri constitue un « monument » curieux qui se caractérise par sa grande hétérogénéité, mêlant notices biographiques aux noms propres de pays ou de villes, aux noms d'ordres religieux ou de monnaies auxquels le classement alphabétique doit conférer un ordre (Foucault 1966 : 53). En publiant ce vaste « dépôt de la gloire » de matériaux puisés dans les nombreux recueils, galeries, dictionnaires ou autres généalogies, l'auteur cherche non seulement à proposer un tableau de l'Histoire universelle, des origines mythologiques et bibliques à l'époque moderne, mais encore d'offrir un catalogue exhaustif des Hommes illustres dont les trajectoires doivent, dans une perspective pédagogique et morale, servir de modèles d'action et de vertu (Bruter 1997). Se situant aussi bien dans l'héritage de Boccace (Cerquiglini-Toulet 200I : 630-643) et de Plutarque $^{6}$ que dans la continuité des nombreuses Vies (Histoire des vies 1996), Portraits $^{7}$ ou Galeries (Margaret MacGowan I985 : 4II-422), des Hommes il-

3 Parayre [éd.], Le Grand dictionnaire historique, ou le Mélange curieux de l'histoire sacrée et profane... par le Sr Louys Moreri,... [2 vol., in- $\mathrm{f}^{\circ}$, frontispice et portrait gravé], Lyon, J. Gyrin et B. Rivière, I68I, I683, I687 [2 $2^{\mathrm{e}}, 3^{\mathrm{e}}, 4^{\mathrm{e}}$ édition] ; Parayre [éd.], Le Grand dictionnaire historique, ou le Mélange curieux de l'histoire sacrée et profane... par le Sr Louys Moreri [2 vol., in- $\mathrm{f}^{\circ}$, figures, frontispice et portrait gravé], Lyon, J.-B. de Ville, I688 [ $5^{\mathrm{e}}$ édition] ; Jean Le Clerc [éd.], Le Grand dictionnaire, ou Mélange curieux de l'histoire sacrée et profane... [2 vol. in-f으, Lyon \& Paris, I69I [6 $6^{\mathrm{e}}$ édition].

4 Jean Le Clerc [éd.], Le Grand dictionnaire historique, ou le Mélange curieux de l'histoire sacrée et profane... par le Sr. Louys Moreri, ... [4 parties en 2 vol., in- $\mathrm{f}^{\circ}$; frontispice gravé], Amsterdam, Boom et Van Someren, I694 [6 ${ }^{\mathrm{e}}$ éd.] ; Jean Le Clerc [éd.], Le Grand dictionnaire historique, ou le Mélange curieux de l'histoire sacrée et profane... par le Sr. Louys Moreri,... $6^{\mathrm{e}}$ édition, revue... [4 vol., in- $\mathrm{f}^{\circ}$; frontispice gravé], Amsterdam (\& Utrecht \& La Haye), G. Gallet, I698 ; Jean LE CLERC [éd.], Le grand dictionnaire historique, ou le Mélange curieux de l'histoire sacrée et profane qui contient en abrégé les vies et les actions remarquables... par $M^{\text {re }}$ Louys Moreri... Neuvième édition où l'on a mis le Supplément dans le même ordre alphabétique, corrigé les fautes censurées dans le Dictionnaire critique de Mr Bayle [2 vol., in- $\mathrm{f}^{\circ}$; t. I [A-G] (572 p.) ; t. 2 [G-Z] (588 p.) ; frontispice gravé ; préface pour la $9^{e}$ édition par Jean Le Clerc], Amsterdam \& Utrecht \& La Haye), Aux dépens de la Compagnie, I702.

5 Abbé de Saint-Ussan, Supplément, ou Troisième volume du Grand dictionnaire historique... de L. Moreri [in- $\mathrm{f}^{\circ}$; pièces liminaires; I238 p.], Paris, D. Thierry, I689.

6 Les Vies parallèles traduites par Amyot sont rééditées dix fois au cours du seul Xvie siècle et les rééditions en latin sont encore plus nombreuses. Cf. Patricia Eichel-Lojkine (200I) ; François Hartog (2005); Marianne Pade (2007).

7 Cf. les publications de Gabriel Michel de la Rochemaillet (I562-I642): Pourtraictz de plusieurs hommes illustres qui ont fleuri en France depuis l'an 1500 jusques à présent [gravés par Léonard Gaultier]. - Brefs éloges des hommes illustres desquels les pourtraictz sont ici représentés Paris, J. Le Clerc, s.d.; La Vie des graves et illustres personnages qui ont diversement excellé en ce royaume, sous les règnes de Louis XII, François I I', Henry II, François II, Charles IX, Henry III et Henry IIII heureu- 
lustres publiée au XVI ${ }^{\mathrm{e}}$ et XVII ${ }^{\mathrm{e}}$ siècle, le dictionnaire historique de Moréri subit de profondes transformations entre I68I et I759, les différents rédacteurs chargés des rééditions corrigeant et rajoutant toujours de nouvelles notices biographiques. Entreprise commerciale, le Moréri doit en effet répondre aux attentes d'un public encore restreint et choisi qui devient rapidement la cible d'opérations concurrentes comme celle menée par le Dictionnaire historique et critique de Pierre Bayle (I647-I706) publié en I697 et réédité dès $1702^{8}$. Les corrections et ajouts apportés par le dictionnaire de Bayle justifient rapidement les rééditions successives du dictionnaire, engageant ainsi un processus de concurrence intellectuelle et commerciale entre libraires ${ }^{9}$. Progressivement, ces dictionnaires historiques s'imposent dans le paysage éditorial et culturel d'autant qu'ils semblent rapporter d'importants gains financiers comme peut l'illustrer la fortune réalisée en partie grâce aux trois rééditions (1707, I714, I718) ${ }^{10}$ du dictionnaire Moréri par l'imprimeur parisien Élie Jean-Baptiste Coignard (I667-I735), déjà renommé pour avoir publié la première édition du Dictionnaire de l'Académie française (I694) ${ }^{\mathrm{II}}$. Dans la concurrence qu'ils se livrent, les libraires (parisiens et hollandais) cherchent non seulement à faire prévaloir la qualité et l'exactitude des informations contenues dans leurs ouvrages, mais encore jouent sur la quantité des notices dont le nombre ne cesse d'augmenter, un essor quantitatif dont les effets ne sont pas minces sur la transformation progressive des régimes de réputation. Au fil des rééditions, les successeurs de Moréri adaptent en effet l'entreprise aux nouveaux regards portés sur l'Homme illustre, et participent ainsi, en accordant une place de plus en plus grande aux hommes de mérite, issus du monde des Lettres et des Arts, au bouleversement du régime des réputations dans la société d'ordre du XVIII ${ }^{\mathrm{e}}$ siècle. En plaçant les Hommes de mérite aux côtés des Héros mythologiques et bibliques,

sement régnant, Rouen, J. Petit, I609.

8 Selon Pierre Rétat, "dans la première moitié du XVIII ${ }^{\mathrm{e}}$ siècle, une édition moyenne pour un grand dictionnaire ne dépasse guère le tirage de 2 ooo exemplaires " (Histoire de l'édition française $1983: 234)$.

9 Pierre Bayle utilise plus d'un quart de ses articles pour corriger quelque i6oo «fautes » dans le dictionnaire de Moréri.

Io Vaultier [éd.] \& Père Ange [coll.], Le Grand dictionnaire historique, ou le Mélange curieux de l'histoire sacrée et profane..., Nouvelle et dernière édition revue, corrigée et augmentée [4 vol. in$\mathrm{f}^{\circ}$; frontispice et portrait gravés], Paris, J.-B. Coignard, I707 ; Louis Ellies Du Pin, Supplément aux anciennes éditions du Dictionnaire historique de Moréri, tiré de l'édition de l'an I7I2 [in-f; IV+936 p.], Paris, J.-B. Coignard, I7I4 ; Louis Ellies Du Pin [éd.; pseudonyme de l'abbé de Clairval], Abbé Le Cointe [coll.], Le Grand dictionnaire historique, ou le Mélange curieux de l'histoire sacrée et profane... enrichi de remarques... tirées... du Dictionnaire... critique de M. Bayle, par $\mathrm{M}^{\mathrm{re}}$ Louis Moreri,... Nouvelle... édition [in- $\mathrm{f}^{\circ}(40 \mathrm{~cm}), 5$ vol. (tome I: A-B; t. 2: C-E; t. 3: F-L; t. 4: M-Q; t. 5: S-Z); frontispice et portrait gravé], Paris, J.-B. Coignard, I7I8.

II Par contrat du I ${ }^{\mathrm{er}}$ mars I700, il est associé pour moitié avec Denis Mariette pour la réédition du Dictionnaire historique de Moréri dont le droit était jusqu'alors détenu par les libraires lyonnais (Frédéric Barbier et al. 2007 : I, 489-494). 
des Princes et des Grands, le classement alphabétique crée des voisinages qui, sans les supprimer totalement, tendent à déstabiliser les hiérarchies et les distinctions considérées comme «naturelles». Ainsi, le dictionnaire de Moréri a sans doute joué un rôle dans le processus d'ennoblissement des hommes de Lettres et Artistes et, à l'inverse, dans le déplacement des fondements de la consécration nobiliaire vers des modèles issus du monde des Lettres et des Arts. Or, au tournant des années I750, de nouvelles entreprises apparaissent mettant au jour les transformations du monde de l'imprimé et les enjeux d'un nouveau régime de la renommée lié à un nouveau marché de la biographie. Les grandes entreprises éditoriales menées dans le cadre de la réédition du dictionnaire de Moréri sont en effet progressivement concurrencées par d'autres productions dont les auteurs cherchent à conquérir un nouveau public et à conférer aux dictionnaires une fonction nouvelle. Ce déplacement peut être illustré par le Dictionnaire portatif publié en deux volumes en 1752 par Jean-Baptiste Ladvocat (I709-1765) ${ }^{12}$.

\section{Ladvocat et le statut d'auteur par le dictionnaire}

Après des études au collège jésuite de Pont-à-Mousson, il rejoint la Sorbonne où il devient docteur grâce à la protection du duc d'Orléans auquel il dédicace son ouvrage ${ }^{13}$. Jean-Baptiste Ladvocat n'appartient pas au monde traditionnel des «petites mains » des dictionnaires historiques qui se recrutent, depuis Moréri, au sein du Clergé. Sa position institutionnelle lui donne néanmoins l'opportunité d'être proche des dépôts lui permettant d'avoir accès aux matériaux nécessaires à l'écriture d'un dictionnaire historique. Il est alors rappelé dans son diocèse et acquiert la cure de Domrémy. Professeur d'hébreu en I740 à la Sorbonne, il en devient le bibliothécaire en I742, une position privilégiée qui lui permet d'accéder facilement aux ouvrages les plus divers. Dès la fin des années I740, il investit le monde des dictionnaires, signe du rôle joué désormais par ce genre d'ouvrage pour

I2 Dictionnaire historique portatif contenant l'histoire des patriarches, des princes hébreux, des empereurs, des rois et des grands capitaines, des dieux, des héros de l'antiquité païenne, etc.; des Papes, des ss. Pères, des évêques et des cardinaux célèbres; des historiens, poètes, grammairiens, orateurs, théologiens, jurisconsultes, médecins, philosophes et mathématiciens, etc. Avec leurs principaux ouvrages et leurs meilleures éditions; des Femmes savantes, des peintres, sculpteurs, graveurs, des inventeurs des arts, et généralement de toutes les personnes illustres ou fameuses de tous les siècles et de toutes les nations du monde dans lequel on indique ce qu'il y a de plus curieux et de plus intéressant dans l'Histoire sacrée et profane. Ouvrage utile pour l'intelligence de l'Histoire ancienne et moderne, et pour la connaissance des écrits et des actions des Grands hommes et des Personnes illustres par M. L'Abbé Ladvocat, docteur et bibliothécaire de Sorbonne et professeur de la chaire d'Orléans en Sorbonne, Paris, chez Didot, 1752.

I3 Son père était juge royal des eaux et forêts et subdélégué de Vaucouleurs, dans le diocèse de Toul. 
ceux qui tentent d'accéder à la reconnaissance d'homme de Lettres. Entre I748-I75I, Ladvocat publie, avec Paul-Denis Burtin, la Bibliothèque annuelle et universelle puis en I750, sous le pseudonyme de "Vosgien ", un Dictionnaire géographique portatif, abrégé du Grand dictionnaire géographique d'Antoine-Augustin Bruzen de la Martinière (I662-I746), qui connaît plusieurs rééditions. En I752, il présente son Dictionnaire historique portatif comme un complément du Dictionnaire géographique. Publié au format in-octavo, l'ouvrage tirerait son origine d'un projet de traduire un dictionnaire anglais, imprimé à Londres en I743. Conforme aux règles du genre, ce dictionnaire offre toujours un « recueil des vies de plus de mille personnes illustres ou fameuses, de tout pays, de tout sexe, de toute condition, depuis le commencement du monde jusqu'à cette année $\mathbf{I} 752$ ». Le titre même renvoie à une représentation traditionnelle de l'Histoire et du monde social :

C'est un recueil [...] dans lequel on rapporte, autant que le sujet le peut permettre et qu'on l'a jugé convenable et nécessaire $\mathrm{I}^{\circ}$ le nom et le surnom de la personne illustre, fameuse ou distinguée dont on parle. $2^{\circ}$ Sa qualité, avec quelqu'épithète qui marque cette qualité. $3^{\circ}$ Le jour, l'année et le lieu de sa naissance $4^{\circ}$ Son père ou sa famille $5^{\circ}$ Ses principales actions ou ses emplois $6^{\circ}$ Le jour, l'année et le lieu de sa mort $7^{\circ}$ Ce qui le caractérise davantage, c'est-à-dire, par exemple, si c'est un Roi, un Empereur, un Pape, les principaux événements de son règne, ou de son Pontificat, avec son Prédécesseur et son Successeur; si c'est un grand Capitaine, ses principales batailles gagnées ou perdues ; si c'est un Peintre, un Sculpteur, un Graveur, un Architecte, ses meilleurs Tableaux, Statues, Estampes, Bâtiments [...] $]^{14}$.

Or, tout en revendiquant, en particulier dans le titre, la continuité avec les grands dictionnaires in-folio de Moréri et de Bayle, Ladvocat met l'accent, au fil des préfaces, sur le caractère abrégé et l'aspect portatif de son ouvrage. Ainsi, en $\mathbf{I} 755$, date de sa première réédition, il présente son dictionnaire comme « une espèce d'abrégé d'histoire universelle dans lequel on trouve, par ordre alphabétique, tout ce qu'il y a de plus important, et ce que l'on voudrait avoir principalement retenu. C'est un recueil des vies de plusieurs milliers de personnes illustres ou fameuses de tout pays, de tout sexe, de toute condition, depuis le commencement du Monde jusqu'à cette année I755 »" Publié une année après les premiers volumes de la fameuse Encyclopédie, ce premier dictionnaire portatif semble répondre à un projet essentiel, conquérir un public plus large ${ }^{\mathrm{I} 6}$ en mettant à disposition un objet plus « commode

I4 Dictionnaire historique portatif, op. cit., Paris, Vve Didot, I760, « Avis ».

I5 Ibid., p. ii.

I6 L'auteur précise les contours de son lectorat dans la suite : « il pourra servir $I^{\circ}$ à ceux qui n'ont pas le moyen d'acheter les grands dictionnaires, ou qui n'ont pas le temps de les lire : $2^{\circ}$ à ceux qui veulent porter avec eux un dictionnaire historique à la campagne, ou à la pro- 
et ordinaire ». L'originalité de l'entreprise de Ladvocat dépasse la seule transformation matérielle. La lecture du titre semble indiquer que l'ouvrage tend à englober, de manière exhaustive, l'histoire des hommes illustres ou fameux de toutes les Nations, une ambition qui s'inscrit dans la continuité du projet de Moréri : il s'agit en effet de présenter les vies des patriarches, des princes hébreux, des empereurs, des rois et les grands capitaines, des dieux, des héros de l'antiquité païenne, des Papes, des Pères, des évêques, mais aussi des historiens, des poètes, des mathématiciens, des femmes savantes ou des peintres. Il justifie en particulier le choix de privilégier les individus dont la réputation repose plus sur les actions et le mérite que sur la naissance ou sur les positions qu'ils ont occupées. Il justifie également la décision de donner moins d'importance aux généalogies : «on ne doit pas s'attendre néanmoins d'y trouver [dans le dictionnaire] généralement tous les hommes dont il est quelquefois fait mention dans l'Histoire, ni ceux qui n'ont eu d'autre mérite que leur naissance, ou qui n'ont été distingués que par les places qu'ils ont occupées. Ce serait un travail immense et de peu d'utilité. [...] Nous avons cru aussi devoir omettre les généalogies, et passer sous silence les personnes encore vivantes. Pour peu qu'on y réfléchisse, on s'apercevra aisément que nous avons de bonnes raisons d'en agir ainsi $»^{17}$. Ainsi, derrière l'apparente continuité, l'entreprise de Ladvocat introduit certaines ruptures, en particulier dans son objet et dans les choix des notices. Pour « entrer » dans le dictionnaire, il ne s'agit plus seulement d'être "illustre ", les élus étant choisis pour leur mérite. Dans cette perspective, l'auteur du dictionnaire se présente comme une instance de consécration, puisqu'il décide des entrées et des sorties du dictionnaire. Le Dictionnaire historique portatif accorde ainsi une place plus grande aux savants et aux hommes de lettres qui prennent progressivement le pas sur les militaires et les ecclésiastiques (Didier 1996 : 15).

Cette évolution rend compte également d'une transformation de la fonction même du dictionnaire. S'il s'agit toujours d'un cadre d'écriture de l'histoire, le dictionnaire s'impose progressivement comme un lieu de publication d'une réputation qui dépend du jugement de l'auteur. Dès lors, le dictionnaire historique s'impose, à l'instar de certains périodiques auxquels participe par ailleurs Ladovat ${ }^{18}$, comme un espace de critique littéraire

menade $: 3^{\circ}$ aux personnes qui sont bien aise d'avoir sous la main un livre commode et d'un usage facile, qui leur rappelle sur le champ les principaux faits et les dates de ces faits $4^{\circ}$ aux personnes du sexe et aux jeunes gens, lesquels trouveront dans ce petit dictionnaire une esquisse de l'Histoire universelle, avec les vies et les caractères des hommes qui se sont le plus distingués dans tous les siècles : $5^{\circ}$ enfin à ceux qui enseignent l'histoire à la jeunesse ; lesquels pourront facilement, à l'aide de ce livre, inculquer à leurs disciples les faits les plus remarquables, et qu'il est plus important de bien retenir ».

17 Ibid.

I8 Sous le pseudonyme de «Vosgien », il collabore en effet à la rédaction des Annales typogra- 
qui répond à une attente, exprimée dès la fin du XVII ${ }^{\mathrm{e}}$ siècle, pour face faire au mouvement d'essor des productions. Dès cette époque apparaissent des listes de noms d'auteurs mêlant, sans aucune différence, les vivants et les morts. Ceux qui construisent ces listes revendiquent à leur tour la position de guides et de critiques, présentant au public une sélection des «meilleurs » ouvrages. À l'instar d'un Adrien Baillet, de nombreux auteurs sollicitent l'intervention d'un critique susceptible de mettre de l'ordre dans les trop nombreuses productions et de tracer la voie du jugement littéraire et esthétique (Waquet I988: I57-I74) ${ }^{19}$. C'est dans ce contexte qu'émergent des périodiques présentés comme des nouveaux lieux de la critique littéraire. C'est le cas de la Critique désintéressée des journaux littéraires et des ouvrages des savants, par une société de gens de lettres ${ }^{20}$ :

On se plaint de tous côtés que la République des lettres tombe en décadence : nous avons reconnu la justice de ces plaintes et après de sérieuses réflexions, nous avons tâché de nous en garantir par une étude opiniâtre. [...] Qu'on examine les choses de près, on reconnaîtra aisément que la plupart des livres nouveaux ne sont tels que par un titre frappant et trompeur. Ainsi les bibliothèques des curieux se remplissent insensiblement d'ouvrages au-dessous du médiocre. [...] Maintenant il nous suffit de dire que le public ne saurait être trop reconnaissant envers des Gens de lettres qui entreprennent avec sincérité de réprimer les désordres, qui sont infiniment préjudiciables aux intérêts des particuliers. On peut juger par-là de l'utilité d'un Journal où, sans égard, ni pour les auteurs, ni pour les libraires, on fait connaître par de bonnes raisons les ouvrages qui méritent d'être lus [... $]^{21}$.

Aux côtés de tels périodiques (le Journal des savants est créé en I665), les listes de noms doivent dessiner les contours d'un milieu choisi auquel elles confèrent une unité, une cohérence et une visibilité que celui-ci n'avait pas, participant à cette formalisation du champ littéraire mise en lumière par Alain Viala (1985). Dès lors, les listes des «meilleurs auteurs » se multiplient, jouant un rôle particulièrement important dans la valorisation de la littérature et des auteurs en langue française, célébrant ainsi le « génie national ». Pensons ainsi à cette liste des savants et des écrivains (morts et vivants) publiée par Jean-Baptiste Rocoles (I620-I696), historiographe du Roi, chanoine de Paris, à la suite de la troisième édition de son Introduction

phiques, ou Notice du progrès des connaissances humaines... par une société de gens de lettres [Roux, Morin d'Hérouville, Goulin, l'abbé Ladvocat et autres], Paris, I760-1763, IO vol.

I9 Cf. Adrien Baillet, Jugements des savants sur les principaux ouvrages des auteurs, Paris, I722, 7 vol.

20 Critique désintéressée des journaux littéraires et des ouvrages des savants, par une société de gens de lettres..., La Haye, chez Chrétien Van Lom, janv.-sept. 1730 (I-III).

2I Ibid., tome I. Préface. 
générale à l'histoire ${ }^{22}$. Le lien, manifeste depuis Pierre Bayle, rédacteur des Nouvelles de la République des lettres (1684-1718), entre la rédaction des périodiques et celle des dictionnaires se renforce, les deux pratiques s'imposant comme des lieux essentiels de l'émergence d'un espace critique ${ }^{23}$. C'est en effet la réputation construite par le biais de l'écriture qui est privilégiée par Ladvocat qui justifie ainsi la place plus grande accordée aux personnages «médiocres »:

Nous avons été tentés d'élaguer ce qu'il y en avait dans ce Dictionnaire et de nous en tenir aux personnages qui jouissent d'un grand nom par eux ou par leurs ouvrages [...] mais nous avons été arrêtés par une forte considération, c'est que les personnages les plus fameux sont les plus connus, et ceux que l'on cherche moins dans un Dictionnaire. C'est au contraire les personnages médiocres, dont on trouve peu de choses dans les Livres que l'on a coutume de lire, que l'on cherche à connaître dans les Dictionnaires. Nous n'avons donc rien supprimé ${ }^{24}$.

Cette évolution justifie encore des mutations dans l'écriture des différentes notices. Pour les personnalités « modernes » qui entrent dans le dictionnaire, la notice biographique accorde en effet une place de plus en plus grande à la bibliographie et à la présentation des ouvrages. Pour Dinah Ribard, " ce qui est remarquable ici, ce n'est pas seulement l'affirmation de la fonction auteur, c'est la réduction de la catégorie des savants à celle des auteurs, c'est-à-dire d'une qualité à une production » (2003: 276). Progressivement, la bibliographie semble même s'imposer comme le critère essentiel qui justifie la présence dans le dictionnaire : «Desmarets (Nicolas), neveu de Colbert et ministre d'État sous le règne de Louis XIV puis Contrôleur général des Finances, a laissé un mémoire très curieux sur son administration. Ce mémoire a été imprimé plusieurs fois. Il est m. en I72I $»^{25}$. L'ouvrage offre ainsi un vaste panorama des auteurs et de leurs productions à un moment où les statuts même d'auteurs ou de "gens de lettres " sont l'objet de débats. Le dictionnaire historique de Ladvocat met en cause les modalités traditionnelles de publication du nom propre et tendent à rendre transparent le rapport entre le nom de l'auteur et ses productions, rapport qui reste alors l'objet de nombreuses stratégies de camouflage ou de brouillage chez

22 Jean-Baptiste Rocoles, Introduction générale à l'histoire, 3 édit., Paris, Denis Béchet, I664, 2 vol.

23 Ainsi Jean Le Clerc (I686-I782), maître d'œuvre de la réédition du dictionnaire de Moréri, rédige la Bibliothèque choisie pour servir de suite à la Bibliothèque universelle, Amsterdam, $\mathrm{H}$. Schelte, I703-I719.

24 Dictionnaire historique et bibliographique portatif par M. l'Abbé Ladvocat, nouvelle édition corrigée et augmentée, Paris, Le Clerc, I777, tome I, Avertissement, pp. xx-xxi.

25 Jean-Baptiste Ladvocat, Dictionnaire historique portatif, Paris, I777, vol. I, p. 518. 
ceux qui revendiquent le statut d'hommes de lettres (Sandrier 2004; Cavaillé 2002 ${ }^{26}$. On peut voir dans ce processus comment le nom propre s'impose progressivement comme le fondement de la «fonction auteur ». Il confère une unité aux productions et s'impose désor mais comme une marque de reconnaissance et de réputation ${ }^{27}$. C'est à partir du moment où le nom propre cristallise des enjeux essentiels dans les dynamiques de construction et de publication de la réputation littéraire qu'il devient le cadre de questionnements nouveaux. Les questions posées par Rousseau sur les usages possibles de «Jean-Jacques » et son apparente paranoïa, les interrogations nouvelles sur l'écriture auto-biographique et sur les relations entre auteur et lecteurs, constituent autant de témoignages des mutations internes à la République des lettres liées à cette émergence du nom propre ${ }^{28}$.

Dans l'article «Gens de Lettres » de l'Encyclopédie (I75I), Voltaire distingue encore le statut d'homme de Lettres de celui de l'auteur et récuse l'assimilation de l'activité des hommes de lettres à une profession, un processus auquel participe le dictionnaire de Ladvocat : «il y a beaucoup de gens de lettres qui ne sont point auteurs, et ce sont probablement les plus heureux ; ils sont à l'abri des dégoûts que la profession d'auteur entraîne quelquefois, des querelles que la rivalité fait naître, des animosités de parti, et des faux jugements; ils sont plus unis entre eux ; ils jouissent plus de la société ; ils sont juges; et les autres sont jugés » (Vovelle 1996 : I63). En I759, Malesherbes, dans la continuité de ces critiques exprimées par Voltaire, rappelait dans son second mémoire sur la librairie : «Il y a quantité d'auteurs qui ne veulent pas être connus, quoique leur ouvrage ne contienne rien de répréhensible " (Malesherbes 1969 [1809] : 37). Il met à l'écart autant la dépendance créée par le mécénat aristocratique que les contraintes du marché contrôlé par les libraires et le public. Or, comme l'ont montré de nombreuses études, le poids des auteurs sans " profession » ou état augmente dans les différentes recensions offertes par la France littéraire ou par les rapports de police rédigés par l'inspecteur de la librairie d'Hemery sur le monde des auteurs parisiens (Darnton 20II : 199-252). Certains, comme Rémond de Saint-Sauveur en I755, peuvent même prétendre enseigner les

26 Rappelons ainsi que les «pauvres diables » dénoncés par Voltaire sont justement les auteurs qui décident de publier sous leur nom. Cf. Henri Duranton (dir.), Le Pauvre Diable. Destins de l'homme de lettres au XVIII siècle, Saint-Étienne, Publications de l'Université de SaintÉtienne, 2006.

27 Un nom d'auteur assure une fonction classificatoire en permettant de regrouper un certain nombre de textes, de les délimiter et de les distinguer d'autres ensembles; le nom de l'auteur effectue en outre une mise en rapport des textes entre eux (Michel Foucault 1994 : I 790-82I).

28 Sur le fameux « Rousseau juge de Jean-Jacques », voir Lilti $2010: 77-94$. Voir également Darnton 2OII : 2OI-234; Goulemot \& Masseau I994 : I-I2. 
astuces et les ficelles pour devenir un écrivain ! ${ }^{29}$ 'est ce mouvement que l'entreprise menée par Ladvocat tente d'enregistrer.

La réputation que légitime ainsi le dictionnaire est celle fondée sur les ouvrages, littéraires ou non, signe indéniable de la promotion de la figure de l'auteur (Jouhaud 2000). En donnant ainsi à son dictionnaire la fonction de publier la réputation des auteurs, Ladvocat s'érige en critique dont la fonction n'est plus seulement d'écrire l'Histoire mais de diffuser des jugements sur les diverses productions, justifiant ainsi les ajouts des notices lors de la réédition de 1755 . À cette date, Ladvocat justifie cette inflation quantitative des noms : " J'ai profité des remarques qui m’ont été envoyées, et l'on trouvera dans cette nouvelle édition plus de cinq cent articles qui avaient été omis dans la première, soit par oubli, soit parce que je n'avais pas alors de Mémoires exacts. On y verra en particulier les articles de Swift, de Schultens, de Noldius, de Ramsay, du Cardinal Valerio, de Grégoire de S. Vincent, de Volkelius, de Don Louis de Haro, du célèbre Boysleve, premier prévôt de Paris, etc. $\aleph^{30}$. On peut d'ailleurs penser que son dictionnaire participe à sa promotion, sous l'égide de Malesherbes, comme censeur royal en I755 (Sgard I999 : 443), année pendant laquelle il en publie une deuxième édition. Dans cette fonction, il se spécialise particulièrement dans l'évaluation des livres protestants dont il se fait le protecteur en prônant une certaine tolérance à leur égard (Birn 2007: II7).

Dans la continuité des recueils du $\mathrm{XVI}^{\mathrm{e}}$ siècle, le dictionnaire historique s'impose comme un espace majeur dans les dynamiques de la République des Lettres. Il constitue progressivement un lieu d'écriture de l'histoire littéraire, de diffusion des normes et du modèle de l'homme de Lettres (RHLF I972 : 284-246 ; Jouhaud I988 : 849-866). D'autres productions tentent progressivement à faire leur apparition sur ce terrain. C'est en particulier le cas du Dictionnaire historique ou Mémoires critiques et littéraires de Prosper Marchand (I676-I758), ouvrage posthume. Ce travail érudit a pour objectif de dresser un tableau de la République des lettres. Prosper Marchand est une des grandes figures du monde de la Librairie du premier XVIII ${ }^{\mathrm{e}}$ siècle. Ce fils d'un musicien du Roi, né à Saint-Germain-en-Laye, est reçu libraire en août I698. Installé à Paris (rue Saint-Jacques, vis-à-vis la fontaine Saint-Séverin), il doit quitter le royaume pour fuir la persécution contre les protestants et se réfugie à La Haye à partir de I709 (Jacob I98I : I82-2I4 ; Berkvens-Stevelink I987). Il abandonne la profession de libraire en I7I3 et devient correcteur chez Fritsch et Böhm à Rotterdam entre I7I7-I723 puis collabore à la rédaction du Journal littéraire (I7I3-I737). Éditeur, auteur de catalogues de librairie,

29 Remond de Saint-Sauveur, Agenda des auteurs ou Calepin littéraire à l'usage de ceux qui veulent faire des livres. Ouvrage didactique pour le dix-huitième siècle, Au Parnasse, d'Anonyme Fertile, imprimeur ordinaire d'Apollon, I755.

30 Dictionnaire historico-portatif..., Paris, chez Didot, « Avis pour cette nouvelle édition », I755. 
ses relations avec des personnalités de la République des lettres en France, comme en Europe, avec qui il entretient une correspondance particulièrement dense (SVEC I993; I984), lui fournissent les outils nécessaires à la publication de son Dictionnaire. Il se charge d'ailleurs de diriger, en I720, la troisième édition du Dictionnaire historique et critique, par M. Pierre Bayle ${ }^{31}$. Dans la continuité de l'entreprise conduite par Ladvocat, la préface rédigée par l'éditeur insiste sur le fait que Marchand a mené son entreprise dans ses moments de « loisirs » et que l'objectif recherché est de guider un public confronté à des productions de plus en plus nombreuses ${ }^{32}$. Ouvrage d'histoire des Lettres, ce dictionnaire se présente encore comme un lieu de diffusion des normes littéraires. Progressivement, les dictionnaires, à travers la concurrence qui les oppose, construisent un espace de débats et de critique au sein du monde des Lettres. Les rééditions orchestrées par Ladvocat (I755 puis $176 \mathrm{O}^{33}$ ) peuvent se justifier, autant par le succès possible de l'ouvrage, que par la volonté de l'auteur de suivre l'actualité nécrologique et d'enrichir un ouvrage dont la fonction est de proposer une histoire des auteurs la plus récente.

Le phénomène dépasse largement les frontières du royaume de France et s'étend partout en Europe, particulièrement dans les États germaniques qui produiraient, selon un correspondant de la Bibliothèque germanique en

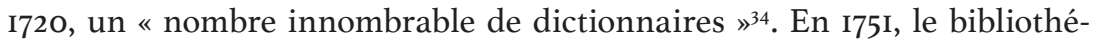
caire de l'Université de Leipzig, Christian Gottlieb Jöcher (I694-I758), publie un Dictionnaire universel des savants ${ }^{35}$. Cet ouvrage, qui sera réédité et enrichi jusqu'au début du XIX ${ }^{\mathrm{e}}$ siècle ${ }^{36}$, vise à collecter les noms de tous les " savants » en Europe et s'impose dès lors comme un cadre majeur de construction de la « République des lettres ». Comme le souligne D’Alembert dans l'Encyclopédie, l'auteur d'un dictionnaire historique doit faire œuvre de critique, la

3I Dictionnaire historique et critique, par M. Pierre Bayle, Rotterdam, M. Bohm, I720, 4 vol., infol.

32 Dictionnaire historique ou Mémoires critiques et littéraires concernant la vie et les ouvrages de divers personnages distingués particulièrement dans la République des lettres, par Prosper Marchand, Paris, P. De Hondt, 1758.

33 Dictionnaire historique portatif, contenant l'histoire des patriarches, des princes hébreux, des empereurs, des rois et des grands capitaines, des dieux, des héros de l'antiquité païenne... des Papes, des SS. Pères, des évêques, ... Paris, Vve Didot, I760.

34 Bibliothèque germanique, I720, vol. II, p. 197.

35 Allgemeines Gelehrten-Lexikon, darinne die Gelehrten aller Stände sowohl männ- als weiblichen Geschlechts, welche vom Anfange der Welt bis auf jetzige Zeit gelebt, und sich der gelehrten Welt bekannt gemacht, nach ihrer Geburt, Leben, merkwürdigen Geschichten, Absterben und Schriften aus den glaubwürdigsten Scribenten in alphabetischer Ordnung beschrieben werden, Leipzig, J. F. Gleditschen, I75I, 4 vol.

36 Dans les années 1780, l'ouvrage est continué par Jean-Christophe Adelung (I732-18o6), bibliothécaire de l'électeur de Saxe ; puis encore continué dans les années I8Io par Heinrich Wilhem Rotermund : Fortsetzung und Ergänzungen zu Christian Gottlieb Jöchers Allgemeinem Gelehrten-Lexicon... Leipzig, J.-F. Gleditschens Handlung, I784-I8I9, 6 vol. 
notice biographique se transformant en jugement porté sur des productions littéraires ou non. Dès lors, de nouvelles normes d'écriture de ces notices sont mises en place, faisant ainsi évoluer la fonction du dictionnaire :

En proscrivant la satyre, on ne saurait au contraire trop recommander la critique dans un dictionnaire littéraire ; c'est le moyen de le rendre instructif \& intéressant : mais il faut que cette critique soit raisonnée, sérieuse \& impartiale ; qu'elle approuve \& censure à propos, \& jamais d'une manière vague; qu'elle ne s'exerce enfin que sur des ouvrages qui en vaillent la peine, \& que par conséquent elle soit pleine de politesse \& d'égards. Cette manière de critiquer est la plus difficile, \& par conséquent la plus rare; mais elle est la seule qui survive à ses auteurs. Une discussion fine \& délicate est plus utile, \& plus agréable même aux bons esprits, qu'une ironie souvent déplacée ${ }^{37}$.

Le dictionnaire de Ladvocat peut être ainsi considéré comme une étape majeure pour comprendre « comment l'auteur s'est individualisé [...], quel statut on lui a donné, à partir de quel moment [...] on s'est mis à faire des recherches d'authenticité et d'attribution, dans quel système de valorisation l'auteur a été pris, à quel moment on a commencé à raconter la vie non plus des héros mais des auteurs » (Foucault 1994: 790). À travers les transformations qu'il fait subir au dictionnaire, Ladvocat parvient à s'imposer lui-même comme auteur ${ }^{38}$. Ainsi, rédiger ou collaborer à la réédition d'un des « grands » dictionnaires devient désormais un moyen de faire carrière dans les Lettres. Selon Dinah Ribard, «la rédaction d'un Dictionnaire historique apparaît donc vers le milieu du XvIII ${ }^{\mathrm{e}}$ siècle à ceux-là même qui s'y attellent comme une contribution à l'élaboration d'une histoire du groupe auquel ils appartiennent principalement, celui des auteurs » (2003: 290). Dans ses différentes préfaces, Ladvocat ne cesse d'ailleurs de mettre en scène un statut d'auteur, distinct de l'érudit, dont le travail de rédaction du dictionnaire ne relèverait que du loisir : « comme nous n'avons fait cet ouvrage que dans nos temps d'amusements et dans les courts intervalles que nous laissent des études sérieuses et des occupations plus importantes, s'il avait fallu ne rien dire que de nous-mêmes, cela nous aurait demandé un temps assez considérable que nous sommes obligés d'employer à des matières plus graves et conformes à notre état $»^{39}$. Si c'est justement sur cette représentation fondée sur le loisir que l'entreprise sera attaquée, il reste qu'à la fin des années $\mathbf{1 7 6 0}$, le dictionnaire de Ladvocat est indéniablement parvenu à s'imposer sur le marché français. Alors que le Grand dictionnaire de

37 Jean Le Rond D’Alembert, «Dictionnaire», consultable en ligne, op. cit.

38 Ladvocat entre ainsi lui-même dans l'édition de I777 de son dictionnaire.

39 Dictionnaire ... par M. l'Abbé Ladvocat, nouvelle édition corrigée et augmentée, Paris, Le Clerc, I777, tome I, « Avertissement », p. ix. 
Moréri est réédité une dernière fois en I759, la troisième édition française du dictionnaire de Ladvocat donne lieu en I769, à une traduction en russe. L'ouvrage est encore traduit en italien en $1773^{4 \circ}$. Les traducteurs transforment ces ouvrages en les enrichissant des noms des célébrités nationales ${ }^{41}$ et participent progressivement à dessiner les contours d'une République des Lettres à l'échelle européenne. Néanmoins, si le dictionnaire de Ladvocat bénéficie indéniablement d'une audience importante, il convient encore de souligner que l'ouvrage a été l'objet de violentes attaques et polémiques qui soulignent le rôle également essentiel que jouent les dictionnaires historiques dans les dynamiques religieuses au XVIII ${ }^{\mathrm{e}}$ siècle.

\section{Dictionnaires et conflits religieux}

Depuis la fin du XVII ${ }^{\mathrm{e}}$ siècle, les ecclésiastiques sont les «petites mains » des dictionnaires historiques ou non, l'équipe réunie autour de l'Encyclopédie constituant une véritable originalité. Les ordres et les institutions religieuses offrent en effet les opportunités et les matériaux nécessaires à mettre en place des vastes entreprises. Or, ces productions s'imposent, dès l'origine, comme des outils de polémiques et de conflits au sein même du monde religieux ${ }^{42}$. C'est d'ailleurs dans cette perspective que s'inscrit la publication dès I697 du Dictionnaire historique et critique de Pierre Bayle dont la seconde édition, en I702, marque indéniablement une étape importante dans le renforcement du rôle joué par ces productions dans les dynamiques religieuses ${ }^{43}$. Il ne s'agit pas en effet seulement de corriger les « erreurs » de Moréri, mais de dénoncer l'intolérance catholique dont ferait preuve ce dernier et d'ériger un « monument » en faveur des réformés (Rétat I97I ; Mori

40 Dizionario storico portatile... composto in francese dal signor abate Ladvocat, ... Edizione novissima... col supplemento... di Giangiuseppe Origlia,... e colle note del P. D. Anton Maria Lugo, Bassano, a spese Remondini di Venezia, I773, 7 t. en I vol. Gian Giuseppe Origlia, le P. Anton Maria Lugo, Palazzi, Abbé Antonio Palazzi, Francesco Antonio Zaccaria.

4I Le traducteur Piotr Semionov ajoute ainsi une vingtaine de noms russes dans le premier volume. Dans l'avertissement, il précise : «En ce qui concerne nos souverains russes, lesquels, Pierre le Grand excepté, ne se trouvent pratiquement pas représentés dans l'original, je n'ai pas manqué, pour ceux qui relèvent de ce premier volume, de les y intégrer, en me conformant constamment à la brève chronique établie par un homme illustre, célèbre dans la république des savants, M. le professeur et conseiller d'État Lomonossov (il s'agit de son Abrégé des Annales de Russie avec une généalogie des souverains, paru en I760), et j'ai l'intention de m'y conformer dans les volumes qui ne manqueront pas de suivre, s'il n'y en a pas de plus complète » (Zaborov 2006 : I89).

42 Dans l'article consacré à " Machiavel ", Louis Moréri s'attaque ainsi au Prince et soutient l'Anti-Machiavel d'Innocent Gentillet et fait remarquer que « la vraie politique n'a rien de contraire aux règles du christianisme », Le Grand dictionnaire, op. cit., I759, t. VIII, p. I7.

43 Dictionnaire historique et critique, Amsterdam, chez Reinier Leers, I697, 4 parties en 2 vol. (in-folio). 
I999; Bost \& de Robert 1999). Dans les années I720-I730, les jansénistes investissent à leur tour le genre du dictionnaire historique dont les notices deviennent le cadre d'opérations de promotion ou de stigmatisation par le biais desquelles s'écrit l'histoire et se construit l'identité des groupes qui s'opposent. La réédition du dictionnaire de Bayle publiée entre I750 et 1756 par Jacques-Georges Chauffepié (I702-I786) constitue une nouvelle offensive en faveur de la cause protestante ${ }^{44}$. Si les dictionnaires de Bayle et de Moréri sont justement devenus des ouvrages " fondateurs ", c'est parce qu'ils ont été utilisés comme des viviers de matériaux biographiques face auxquels les auteurs postérieurs sont venus piocher, corriger et ajouter des éléments nouveaux, répondant aux exigences des combats qu'ils mènent (Geissler 1971 : I2I-I4O). En Suisse, en Hollande ou en France, les rééditions successives de ces « grands » dictionnaires permettent ainsi à plusieurs générations d'entrepreneurs d'afficher leurs partis pris religieux. L'écriture des biographies individuelles des membres de l'Église, mais aussi des hommes de Lettres, est ainsi progressivement mobilisée pour faire triompher l'un des camps. Ces luttes ont pour effet de provoquer un essor du nombre des notices justifiant les rééditions, chacun cherchant, non seulement à dénoncer ses adversaires, mais à lever des armées de papier en faisant entrer des personnalités gagnées à leur cause. On peut penser que les différents dictionnaires sont les centres de vastes réseaux de souscripteurs à partir desquels se dessinent les contours d'un camp : posséder le Moréri ou le Bayle constitue dès lors un marqueur d'appartenance religieuse. Les dictionnaires s'imposent ainsi comme de véritables champs de bataille. Le Dictionnaire portatif de Ladvocat ne reste pas en dehors de ces logiques d'affrontement.

Entre 1758 et I759, Pierre Barral (vers I700-I772), l'un des représentants éminents du jansénisme parlementaire ${ }^{45}$, mène l'offensive contre ses adversaires en publiant, sous l'anonymat, un dictionnaire qui s'impose progres-

44 Cf. Nouveau Dictionnaire historique et critique, pour servir de supplément ou de continuation, au Dictionnaire historique et critique de M. Pierre Bayle par Jacques-Goerges de Chauffepié, Amsterdam, La Haye, Z. Chatelain et fils, H. et M. Uytwerf, J. Weisten, Arkstee et Merkus, M.M. Rey, Pierre de Hondt, I750-1756, 4 vol. in-fol. Ministre et prédicateur calviniste, Chauffepié exerce, entre $\mathrm{I} 745$ et I786, à l'église protestante d'Amsterdam. À la suite de la publication d'une traduction anglaise de l'ouvrage de Bayle, avec des additions considérables, un imprimeur-libraire d'Amsterdam propose à Chauffepié de traduire en français les additions faites en Angleterre. Ces additions consistaient, soit en compléments des articles de Bayle, soit en articles nouveaux, mais privilégiaient surtout les hommes illustres de Grande Bretagne. II consacre plusieurs années à ce travail, et fit lui-même de nouvelles additions et des articles nouveaux. Sur près de quatorze cents articles qu'on trouve dans son dictionnaire, plus de six cents, presque tous anglais, sont traduits sans additions de la part de Chauffepié ; deux cent quatrevingts environ sont retouchés par lui ; cinq cents articles environ sont entièrement de lui.

45 Pierre Barral, Appelants célèbres ou abrégé de la vie des personnes les plus recommandables entre ceux qui ont pris part à l’Appel interjeté contre la bulle Unigenitus, s.l., I753. 
sivement comme un véritable « martyrologe $»^{46}$. Si l'auteur prend nettement position contre les continuateurs de Moréri, il attaque particulièrement le dictionnaire de Ladvocat qui aurait voulu « déprimer les plus célèbres auteurs, en les accusant d'avoir été opposés aux prétendues décisions de l'Église contre Jansénius et le P. Quesnel. [...] Ne doit-on pas être encore plus indigné du silence coupable, qu'il garde sur le cardinal Le Camus, le S. Évêque de Senez [Joan Soanen], l'illustre Boursier, et d'autres grands hommes, qui avaient un droit si bien acquis sur un Dictionnaire, fait pour les célébrer ${ }^{47}$. Barral se propose ainsi de corriger et de compléter l'ouvrage de Ladvocat afin de défendre la cause des jansénistes ${ }^{48}$. Cette initiative est relayée par d'autres entreprises proches de ce courant. En I759, Étienne-François Drouet (I7I5-I779) et l'abbé Claude-Pierre Goujet (I697-I767) s'associent, non pour lancer une nouvelle entreprise, mais pour publier une réédition du dictionnaire de Moréri, la dernière du XVIII siècle. Goujet, particulièrement engagé en faveur des jansénistes, fréquente les premières conférences figuristes dans les années I730. Proche de Le Paige, il est l'une des plumes particulièrement actives dans le combat mené contre les jésuites. En I735 puis I749, après avoir publié Les Vies des saints qui connaîtront une fortune éditoriale jusque dans les années I830, il publie déjà un premier supplément au dictionnaire de Moréri Au cours des années I750, il multiplie les initiatives contre les adversaires des jansénistes ${ }^{49}$. Le fait que son collaborateur, Étienne-François Drouet soit avocat et bibliothécaire au Parlement, membre de l'Académie d'Auxerre et de la Société littéraire de Besançon, montre que ces productions peuvent participer à la construction de l'unité et de la visibilité du « parti » janséniste. Pris en main par les jansénistes, les dictionnaires jouent indéniablement un rôle essentiel dans la mise en forme d'une histoire de Port-Royal selon le modèle d'une "galerie de portraits " ${ }^{50}$. Comme Barral, les rédacteurs de cette réédition prennent pour cible le dictionnaire de Ladvocat, dont une nouvelle mouture était sortie des presses en I755, considéré alors comme le porte-parole des jésuites. Or, dès cette date, Ladvocat tente de mettre à distance ces querelles religieuses et de replacer son entreprise dans le cadre d'activités de loisir liées à la « littérature et à

46 Pierre Barral, Dictionnaire historique, littéraire et critique, contenant une idée abrégée de la vie et des ouvrages des hommes illustres en tout genre, de tout temps et de tout pays..., Avignon, [s.n.], I758-I759, 6 vol.

47 Ibid., préface.

48 Il faut rappeler que Barral joue un rôle central dans la théorisation du jansénisme dit « constitutionnel » (Cottret I998: I69-I72).

49 Observation sur un ouvrage intitulé Dictionnaire des livres jansénistes ou qui favorisent le jansénisme, À Anvers, chez J. B. Verdussen, I752, 4 volumes. En France. I755.

50 Drouet participe à la grande entreprise de rédaction des Mémoires pour servir à l'histoire de Port-Royal, (s.l.), 1734-1737, 3 tomes en 2 vol. 
l'histoire ». Il met en avant son statut d'auteur et de critique contre celui de polémiste :

L'accueil favorable que le public a fait à mon ouvrage, m’a engagé à le revoir avec soin. On y trouvera plus de huit cents articles qui avaient été omis dans les éditions précédentes, soit par oubli, soit parce que je n'avais pas alors de mémoires exacts, ou que les personnes qui sont les sujets de ces articles étaient encore vivantes. [...] Un écrivain anonyme [Barral] a cru mieux faire de publier contre moi un gros Dictionnaire [...] dans lequel il m'accable d'injures, quoique je ne le connaisse point et que je n'aie jamais rien dit ni écrit contre lui. Il va plus loin; il me prête des vues et des intentions que je n'ai jamais eues et auxquelles je n'ai pas même pensé. Il est constant que j'ai composé ce petit ouvrage à la campagne pendant les vacances, par manière de délassement et dans l'unique dessein de donner aux jeunes gens qui me consultent souvent une idée juste de l'histoire et de la littérature. J'y ai apporté l'esprit de modération, de paix, de douceur et d'impartialité que je recommande sans cesse à la jeunesse. [...] Mais l'Auteur des Nouvelles ecclésiastiques et après lui l'écrivain anonyme dont je parle, soit par esprit de parti, soit par d'autres motifs, pensent bien différemment de mon dictionnaire. Ils m'accusent de trop exalter les Jésuites et leurs amis et de déprimer avec excès les célèbres écrivains de Port-Royal, et les autres adversaires des Jésuites ${ }^{51}$.

Si progressivement d'autres productions vont s'imposer dans les luttes entre jansénistes et leurs adversaires, il n'en reste pas moins que les dictionnaires historiques jouent un rôle essentiel dans les combats religieux jusqu'à la fin du XVIII ${ }^{\mathrm{e}}$ siècle. En I769, l'auteur du Dictionnaire historique-portatif des ordres religieux et militaires dénonce, au lendemain de l'expulsion des Jésuites, les offensives menées contre les ordres réguliers ${ }^{52}$. Progressivement, les combats évoluent et les adversaires se transforment. Dans les années I770, les dictionnaires historiques s'imposent comme les instruments majeurs de l'offensive menée contre les philosophes. Puisant toujours leurs matériaux dans les premiers « grands » dictionnaires, ces productions donnent corps au courant des anti-Lumières. En I77I, l'abbé de Bonnegarde publie ainsi une réédition du dictionnaire de Bayle pour défendre le christianisme contre les philosophes :

Il était d'une nécessité indispensable de faire un choix en faisant notre abrégé. Parmi les fruits que nous offraient les vergers abondants où nous

5I Dictionnaire historique-portatif... par M. l'abbé Ladvocat, Docteur, Bibliothécaire et professeur de la Chaire d'Orléans, en Sorbonne, Paris, veuve Didot, I76I, « Avertissement », pp. xiv-xv.

52 Dictionnaire historique-portatif des ordres religieux et militaires et des Congrégations régulières et séculières qui ont existé jusqu’à nos jours contenant leur origine, leur progrès, leur décadence... par monsieur M.C.M.D.P.S.J.D.M.E.G, à Amsterdam, chez Marc-Michel Rey, I769. 
avons puisé, nous n'avons cueilli que les plus agréables, et nous avons écarté ceux qui étaient empoisonnés. Ceux-ci sont en grand nombre dans le dictionnaire de Bayle, l'apôtre le plus dangereux du déisme et du pyrrhonisme. [...] Je n'ai point cherché à prêcher ; mais je me suis fait un devoir d'écarter tout ce qui pouvait blesser la religion. [...] Les incrédules modernes nous en sauront mauvais gré, mais on n'a que trop travaillé pour eux dans deux différents extraits du philosophe de Rotterdam. [...] Bien loin d'imiter ces téméraires abréviateurs, nous avons pris quelquefois la défense du christianisme, lorsque l'occasion s'en est présentée. [...] Quelques articles changés, beaucoup d'autres retranchés l'auraient laissé jouir du calme qu'il méritait à tant d'égards. Comment oserait-on me blâmer d'avoir fait ce qu'il aurait dû faire, et ce qu'il se repentit sans doute de n'avoir pas fait, dans l'agitation et le trouble que lui causèrent les disputes occasionnées par les morceaux que j'ai proscrits? [...] On aurait désiré aussi que l'auteur eût retranché quelques déclamations déplacées contre les catholiques. Cette aigreur de parti n'est ni du goût particulier des gens de lettres, ni du goût général du siècle ${ }^{53}$.

Les années I750 se caractérisent par la place de plus en plus importante prise par les dictionnaires historiques dans l'espace éditorial. Plus généralement, cette montée en puissance des dictionnaires participe à l'explosion des biographies à travers la multiplication des Vies privées, éloges, oraisons ou nécrologes. Daniel Roche a montré que la pratique de l'éloge s’impose et se diffuse dans les différentes académies royales dès la fin du XVII ${ }^{\mathrm{e}}$ siècle (Merlin 200I) et se généralise au cours du XVIII ${ }^{\mathrm{e}}$ siècle dans les diverses académies de province (Roche I978 : I66 ; Paul 1980). Dans l'espace de la peinture, la production des Vies d'artistes devient une part majeure de la contribution des membres honoraires au travail de l'Académie royale de peinture, une pratique d'écriture susceptible de révéler des dispositions particulières (jugement de goût, connaissance visuelle des œuvres...) qui s'imposent comme centrales dans la promotion de la figure de " l'amateur » (Guichard 2008 : 67 ; Waschek 1996). À travers l'écriture de ces Vies se dessinent, selon Charlotte Guichard, les contours d'une École française de peinture, inventée à l'occasion et associée à l'histoire de l'Académie royale. Il s'agit bien d'écrire l'histoire de l'académie et d'imposer, par-là, un régime de réputations et de normes artistiques. Cette perspective se retrouve dans d'autres domaines dans lesquels l'écriture biographique joue encore un rôle majeur. Comme le rappelle encore Dinah Ribard, « la biographie est la forme privilégiée de l'histoire de la philosophie à l'époque moderne » (1978 : 328). Comme pour les arts, les Vies des philosophes deviennent les supports de la normalisation

53 Abbé de Bonnegarde, Dictionnaire historique et critique, ou Recherches sur la vie, le caractère, les mœurs et les opinions de plusieurs hommes célèbres, tirées des Dictionnaires de Mrs Bayle et Chauffepié, ouvrage dans lequel on a recueilli les morceaux les plus agréables et les plus utiles de ces deux auteurs avec un grand nombre d'articles nouveaux et de remarques d'histoire, de critique et de littérature, Lyon, Barret, Paris, Delalain I77I, vol. I, Préface. 
de la philosophie, mais aussi le théâtre où se publie l'histoire des conflits et des luttes du champ philosophique. D'un côté comme de l'autre, les Vies intègrent et mettent en scène certains coups de forces historiographiques qui imposent une écriture patriotique de l'histoire des arts ou de la philosophie. C'est encore dans ce contexte que le genre de l'éloge académique est profondément renouvelé par la réforme du prix d'éloquence de l'Académie française en 1758 : désormais consacré aux « hommes célèbres de la nation ", il se substitue aux éloges funèbres du XVII ${ }^{e}$ siècle et propose une laïcisation de la mémoire nationale tournée désormais vers l'avenir.

Reste que la fonction proprement historiographique des dictionnaires tend à être mise en cause. L’année $\mathbf{1 7 5 9}$, date de la dernière édition du dictionnaire de Moréri, peut être considérée comme un tournant. Les efforts pour maintenir dans les années i76o le modèle d'une histoire collective des Vies sous la forme de recueils sont de plus en plus battus en brèche. Plus généralement, l'écriture biographique comme support de l'écriture de l'histoire semble de plus en plus mobilisée dans des entreprises de formalisation menées par les institutions officielles, en particulier par les académies. Jean-Claude Bonnet a parfaitement décrit le mouvement de mutation que les membres de l'Académie française font subir au genre de l'éloge. Désormais consacré aux " hommes célèbres de la Nation ", l'éloge académique se substitue aux oraisons funèbres du XVII ${ }^{e}$ siècle. Entre 1758 et $\mathrm{I} 788$, seize grands hommes sont célébrés dont deux rois, seize militaires et quatre hommes de lettres ${ }^{54}$. S'inscrivant dans un mouvement commencé sans doute dans d'autres institutions ${ }^{55}$, l'enjeu de ces éloges est, dans le contexte particulier de la guerre de Sept Ans, d'écrire une histoire officielle et patriotique au service de la Monarchie. Il s'agit de reprendre le contrôle de l'écriture de l'histoire des vies mise au service de la propagande monarchique. Dans le domaine des arts également, un nouveau regard est porté sur l'écriture des Vies. Dans son Histoire de l'art chez les anciens publiée en I764, Winckelmann affirme avoir « voulu écrire une histoire de l'art; et non une histoire des artistes» (Pommier 2003 : II7). Selon Édouard Pommier, on passerait ainsi d'une " histoire fragmentée à travers le prisme des biographies à une vision d'ensemble, celle de l'histoire de la peinture $»^{56}$. Cette transformation semble participer d'une mutation plus profonde du régime

54 Après avoir remporté le premier concours consacré à l'éloge du chancelier d'Aguesseau, Antoine Léonard Thomas (I732-I785) s'impose comme l'insurpassable spécialiste du genre au point de remporter le prix cinq années de suite.

55 Daniel Roche a montré que la pratique de l'éloge s'est imposée à la fin du XVII ${ }^{e}$ siècle à l'Académie française, au début du XVIII ${ }^{e}$ siècle à l'Académie des sciences avec Fontenelle et dans la première moitié du XVIII ${ }^{\mathrm{e}}$ siècle à l'Académie des Inscriptions. L'Académie de peinture suivra à son tour ce modèle. Ce modèle a été progressivement suivi par les académies de province (Roche $1978:$ I66).

56 Ibid., p. I4I. 
d'historicité, l'histoire « exemplaire » (ou Historia magistra) (Kosseleck 1990) et répétitive laissant progressivement place à une histoire conçue comme processus (Hartog 2003 : II6-II7). Dans cette perspective, les dictionnaires historiques ne deviendraient-ils pas, dans la seconde moitié du XVIII ${ }^{e}$ siècle, des espaces de conservation de pratiques d'écriture de l'Histoire de plus en plus anachroniques ? Le succès apparent de ces productions s'expliquerait-il par leur rôle pédagogique, les notices biographiques proposant encore des « modèles » à suivre? Dans sa préface de I77I, l'abbé de Bonnegarde s'étonne d'ailleurs de l'intérêt que peuvent susciter des dictionnaires historiques qui, loin désormais de se restreindre aux récits de vies des hommes «illustres ", publient des récits biographiques de personnages ordinaires :

Ce serait peut-être ici le lieu d'examiner pourquoi des articles qui ne semblent renfermer que de petites circonstances sur la vie de quelques hommes inconnus, sont lus avec autant d'avidité par les gens de lettres, et même par ceux qui ne le sont point, que les romans sont recherchés par les femmes. D'où vient cette curiosité à l'égard de quelques hommes qui n'ont joué aucun rôle important sur le théâtre du monde, ou qui du moins n'ont été auteurs ou spectateurs que de quelques pièces bourgeoises dans la comédie de la vie ? [...] Ce qu'on appelle ordinairement des petites choses, des menus faits, des riens personnels ou domestiques, en un mot, toutes les prétendues minuties sont souvent plus intéressantes pour le cœur humain, que les mémoires d'un général ou d'un ministre. Tout hommes est homme, mais tout homme n'est pas général d'armée, ministre d'état ; ou plutôt la plupart des hommes ne sont rien d'approchant. Donc, tout ce qui montrera bien l'homme, attachera plus que ce qui ne montrera que le général, le ministre, le négociateur ou même le savant et l'homme de lettres. [...] C'est l'amour propre qui se cherche et il ne se trouverait point dans les grandes histoires ${ }^{57}$.

Ces dictionnaires seraient donc pourvoyeurs des « petites » histoires susceptibles d'apporter des leçons de vie et des modèles d'actions qui ne sont pas uniquement issus des existences extraordinaires ou héroïques. Conjuguée au classement alphabétique qui efface toutes les formes de hiérarchie, cette place plus grande accordée aux « circonstances sur la vie de quelques hommes inconnus » bouleverse le regard traditionnel porté sur la " grandeur » et contribue à redessiner les nouveaux contours de la "réputation » et de la « renommée ». Sans qu'il soit question de dire que l'organisation du dictionnaire a pour effet de tout niveler, il convient néanmoins de constater qu'il contribue à créer de nouvelles proximités entre des individus aux conditions sociales parfois très éloignées. Être ou ne pas être dans le dictionnaire trace désormais une frontière à partir de laquelle se construisent de nouvelles distinctions. On peut plus généralement constater, à travers

57 Abbé de Bonneguarde, Dictionnaire, op. cit., vol. I, « Préface », pp. xii-xiii. 
l'exemple de l'entreprise menée par Ladvocat, que la fonction des dictionnaires historiques évolue. À partir de I750, il semble que les dictionnaires jouent un rôle dans les mutations qui touchent au régime des réputations individuelles. L'essor des notices biographiques publiées dans ces ouvrages participe en effet au passage progressif entre l'homme « illustre » et l'homme " célèbre ", passage dont témoigne en particulier la transformation du genre de l'éloge à l'Académie française. C'est en effet en 1758 que l'on peut dater " officiellement » la naissance du culte des grands hommes en France, " lorsque les anciens sujets du concours d'éloquence à l'Académie furent remplacés par l'éloge des grands hommes de la nation» (Bonnet 1998 : IO ; $M L N$ 200I : 222-288 $)^{58}$. Les notions de « réputation », « renommée» ou «célébrité » font l'objet d'importants débats autour des années I750, débats dont D'Alembert se fait l'écho dans l'article « Dictionnaire » de l'Encyclopédie. Selon lui, ces dictionnaires doivent comporter l'histoire des "princes", des " grands » et des « hommes célèbres par leurs talents \& par leurs actions ", mais ils doivent intégrer aussi les « hommes illustres dans les Sciences, dans les Arts libéraux, \&, autant qu'il est possible, dans les Arts mécaniques même " ${ }^{59}$. Il convient ainsi de constater un déplacement des fondements de la renommée marqué en particulier par la place plus grande accordée au « mérite", signe, pour certains historiens comme Marc Fumaroli, d'un processus irrémédiable de délitement des fondements sociaux et politiques marquant le passage entre l'âge classique et l'âge moderne (1987 :3-30). Les dictionnaires historiques ne sont pas les seules productions à participer à cette transformation comme en témoigne cet avis des auteurs de L'ordre chronologique des deuils de cour :

[À la suite d'une récapitulation historique des princes morts au cours de l'année sera ajouté] le nécrologue des personnes, célèbres dans les sciences ou dans les arts, mortes dans le même espace de temps, avec un précis de leur vie et de leurs ouvrages. Ce recueil, fait comme il doit l'être, peut fournir un jour des matériaux pour l'histoire de l'esprit humain. Ce n'est qu'après la mort des artistes que l'on ne consulte plus ni l'adulation ni la haine dans les jugements que l'on porte. Ce sera d'ailleurs un nouveau motif ajouté à l'émulation ; ces éloges funèbres étant un tribut qui, chez toutes les nations policées, a été regardé comme un des principaux encouragements du mérite ${ }^{60}$.

58 La décision de l'Académie reprend un projet de l'abbé de Saint-Pierre qui avait proposé que ce corps savant rédigeât une histoire des grands hommes dans le genre des Vies de Plutarque.

59 Jean Le Rond D’Alembert, «Dictionnaire», consultable en ligne, op. cit.

60 L'Ordre chronologique des deuils de Cours avec un précis de la vie et des ouvrages des auteurs qui sont morts dans le cours de l'année, Paris, Moreau, I764. 
Dès lors, se pose la question de la légitimité du dictionnaire et de son auteur comme instances de consécration du renom. Dans la continuité de la citation précédente, D’Alembert n'en rappelle pas moins également la nécessité d'exclure certains individus considérés comme "indignes », en particulier ceux qui, dans le monde des Lettres, ne publient que des satires :

Ce n'est pas néanmoins que l'on doit exclure entièrement d'un dictionnaire les mauvais écrivains ; il est quelquefois nécessaire de connaître au moins le nom de leurs ouvrages : mais leurs articles ne sauraient être trop courts. S'il y a quelques écrivains qu'on doive, pour l'honneur des lettres, bannir entièrement d'un dictionnaire, ce sont les écrivains satyriques, qui pour la plupart sans talent, n'ont pas même souvent le mince avantage de réussir dans ce genre bas \& facile : le mépris doit être leur récompense pendant leur vie, \& l'oubli l'est après leur mort. On a reproché au dictionnaire de Bayle de faire mention d'un assez grand nombre d'auteurs peu connus, \& d'en avoir omis de fort célèbres ${ }^{61}$.

À partir des années 1750 , on peut penser, faute de réelle étude sur cet aspect, que les publics privilégiés des dictionnaires historiques se transforment progressivement. Aux lecteurs restreints et choisis de la première moitié $d u \mathrm{XVIII}^{\mathrm{e}}$ siècle (issus principalement du Clergé et de la Noblesse) semble succéder un public plus large, issu des rangs de la bourgeoisie urbaine, un phénomène qui renvoie aux mutations générales du monde de l'imprimé (Barbier et al. 1996 : 209-225). Or, à partir des années I770, alors que s'est constitué un véritable marché pour ces productions ${ }^{62}$, les dictionnaires sont objets de très nombreuses critiques : accusées de favoriser l'artificialité des connaissances et de ne répondre qu'à l'intérêt des libraires, accusés encore de «brouiller » les hiérarchies « naturelles » et de rabaisser les "Grands » au profit des «médiocres », ces productions semblent participer à la « décadence » des mœurs que les auteurs de ces attaques ne cessent de dénoncer. En I775, les membres de l'Assemblée générale du clergé dénoncent ainsi la «fausse immortalité » des écrivains « incrédules » ${ }^{63}$. Dans

6I Jean Le Rond D’Alembert, « Dictionnaire», consultable en ligne, op. cit.

62 Le Supplément à la France littéraire publié en 1778 (Paris, Veuve Duchesne, t. III, pp. 40-4I) mentionne ainsi 43 dictionnaires. Dans cette liste, 6 appartiennent au genre des dictionnaires historiques : Dictionnaire historique, critique, ou recherches sur la vie, le caractère, les mours et les opinions de plusieurs hommes célèbres, tiré des dictionnaire de Bayle et de Chaufepied, par M. de Bonnegarde, I770 ; Dictionnaire historique des cultes religieux... par M. Lacroix, I770 ; Dictionnaire historique des saints personnages où l'on peut prendre une notion exacte et suffisante de la vie et des actions mémorables des héros du christianisme par M. Lacroix, I770 ; Dictionnaire historique des sièges et des batailles mémorables de l'histoire ancienne et moderne ou anecdotes militaires de tous les peuples du monde, par M. Lacroix, I770 ; Dictionnaire historique et bibliographique portatif... par M. l'abbé Ladvocat, nouvelle édition ... par M. le Clerc, I777 ; Nouveau Dictionnaire historique... par Chaudon, I770.

63 « Vous comprenez déjà, N.T.C.F., que l'immortalité dont nous vous parlons, n'est 
un discours prononcé devant les membres de l'Académie de Montauban en I754 et reproduit dans l'ouvrage de Durey de Noiville, un certain abbé Bellet présente déjà les auteurs de dictionnaires comme des « copistes sans gages qui raconte[nt] seulement ce que les autres ont dit » et ne font qu'un « travail mécanique ${ }^{64}$. Ces critiques, qui ne cessent de se multiplier dans les années I770, semblent mettre en relation le succès des dictionnaires et la crise politique à laquelle la Monarchie est confrontée.

\section{Bibliographie}

Barbier, Frédéric et al. (dir.), Dictionnaire des imprimeurs, libraires et gens $d u$ livre à Paris I707-I787, Genève, Droz, 2007.

Berkvens-Stevelink, Christiane, Prosper Marchand. La vie et l'ouvre (I678I756), Leyde, E.J. Brill, 1987.

Birn, Raymond, La Censure royale des livres dans la France des Lumières, Paris, Odile Jacob, 2007.

Bonnet, Jean-Claude, La Naissance du Panthéon. Essai sur le culte des grands hommes, Paris, Fayard, 1998.

Bost, Hubert \& Robert, Philippe de (dir.), Pierre Bayle, citoyen du monde. De l'enfant de Carla à l'auteur du dictionnaire, Paris, Honoré Champion, 1999.

Cavaillé, Pierre, Dis-simulations : Jules-César Vanini, François La Mothe Le Vayer, Gabriel Naudé, Louis Machon et Torquato Accetto : religion, morale et politique au XVII esiècle, Paris, Honoré Champion, 2002.

Cerquiglini-Toulet, Jacqueline, «À la recherche des pères : la liste des auteurs illustres à la fin du Moyen Âge ", $M L N$, vol. II6, n 4 (sept. 200I), pp. 630-643.

Chartier, Roger, «L'homme de lettres", dans M. Vovelle (dir.), L'homme des Lumières, Paris, Seuil, 1996, pp. 159-209.

Cottret, Monique, Jansénismes et Lumières. Pour un autre XVIII e siècle, Paris, Albin Michel, 1998.

Cristin, Claude, «Aux origines de l'histoire littéraire française : "Les éloges des Hommes sçavants Tirez de l'histoire de M. de Thou par Antoine Teissier" (I683-I715) ", Revue d'histoire littéraire de la France, 22, 1972, pp. 238-246.

-.Aux origines de l'histoire littéraire, Grenoble, Presses universitaires de Grenoble, 1973 .

pas le souvenir que les hommes peuvent laisser d'eux sur la terre après leur mort. Les écrivains incrédules ont essayé quelquefois d'enflammer leurs lecteurs pour cette ombre d'immortalité ", Avertissement de l'Assemblée générale du Clergé de France, tenue à Paris par permission du Roi, en I775 aux fidèles de ce Royaume. Sur les avantages de la religion chrétienne et les effets pernicieux de l'incrédulité, Paris, 1775.

64 Jacques-Bernard Durey de Noinville, Table alphabétique des dictionnaires..., op. cit., pp. 4-II. 
Darnton, Robert, « La République des lettres : les intellectuels dans les dossiers de police », Le Grand Massacre des chats. Attitudes et croyances dans l'ancienne France [1985], Paris, Les Belles Lettres, $201 \mathrm{I}$.

Didier, Béatrice, Alphabet et raison. Le paradoxe des dictionnaires au XVIII siècle, Paris, PUF, 1996.

Duranton, Henri (dir.), Le Pauvre Diable. Destins de l'homme de lettres au XVIII siècle, Saint-Étienne, Publications de l'Université de Saint-Étienne, 2006.

Eichel-Lojkine, Patricia, Le Siècle des grands hommes. Les recueils des Vies d'hommes illustres avec portraits au XVI ${ }^{e}$ siècle, Louvain, Peeters, $200 \mathrm{I}$.

Favre, Robert, "Jean-Baptiste Ladvocat », dans Jean Sgard (dir.), Dictionnaire des journalistes (I660-I789), Oxford, Voltaire Foundation, I999, vol. II, p. 443 .

Foucault, Michel, «Qu'est-ce qu'un auteur ? " [1969], Dits et écrits, Paris, Gallimard, I994, tome I, pp. 790-82I.

Fumaroli, Marc, "Des 'Vies' à la biographie : le crépuscule du Parnasse », Diogène, $\mathrm{n}^{\circ}$ 139, 1987, pp. 3-30.

Geissler, Rolf, " Zu den Fortsetzungen von Bayles "Dictionnaire historique et critique" in der Aufklärung : Die Wörterbücher von Chauffepié und Marchand ", dans W. Bahner (éd.), Beiträge zur Französischen und zur Spanischen Literatur, Berlin, Akamedie-Verlag, I97I, pp. I2I-I40.

Goulemot, Jean-Marie \& Masseau, Didier, « Naissance des lettres adressées à l'écrivain ", Textuel, 27, I994, pp. I-I2.

Guichard, Charlotte, Les Amateurs d'art à Paris au XVIII ${ }^{e}$ siècle, Seyssel, Champ Vallon, 2008.

Hartog, François, «Un ancien chez les modernes : Plutarque », Anciens, modernes, sauvages, Paris, Gallade Éditions, 2005, pp. 99-I47.

—. Régimes d'historicité. Présentisme et expérience du temps, Paris, Seuil, 2003.

Histoire de vies. Actes du colloque de I994, Paris, Presses de l'Université de la Sorbonne, 1996.

Jacob, Margaret C., The Radical Enlightenment: Pantheists, Freemasons and Republicans, Londres-Boston-Sydney, George Allen and Unwin, I98I, pp. I82-2I4.

Jouhaud, Christian, " Histoire et histoire littéraire : naissance de l'écrivain », Annales ESC, 43-4, 1988, pp. 849-866.

-. Les pouvoirs de la littérature. Histoire d'un paradoxe, Paris, Gallimard, NRF essais, 2000.

Kosseleck, Reinhart, Le Futur passé : Contribution à la sémantique des temps historiques, traduit de l'allemand par J. Hoock et M.-C. Hoock, Paris, EHESS, I990.

Lilti, Antoine, "Reconnaissance et célébrité : Jean-Jacques Rousseau et la politique du nom propre », Orages, 9, mars 20I0, pp. 77-94. 
MacGowan, Margaret, "Le phénomène de la galerie des illustres ", dans Roland Mousnier \& Jean Mesnard (éd.), L’âge d’or du mécénat (I598-I66I), Paris, Éditions du CNRS, 1985, pp. 4II-422.

Malesherbes, Mémoires sur la librairie [1809], Genève, Slatkine Reprint, 1969. Merlin, Hélène, L'Excentricité académique. Littérature, institution, société, Paris, Les Belles lettres, 200I.

Mori, Gianluca, Bayle philosophe, Paris, Honoré Champion, 1999.

Pade, Marianne, The Reception of Plutarch's Lives in Fifteenth-Century Italy, Copenhague, Museum Tusculanum Press, 2007.

Paul, Charles B., Science and Immortality. The Eloges of the Paris Academy of Sciences (I699-I79I), Berkeley, University of California Press, I980.

Pommier, Édouard, «Winckelmann : des vies d'artistes à l'histoire de l'art », Winckelmann, inventeur de l'histoire de l'art, Paris, Gallimard, 2003.

Rétat, Pierre, Le Dictionnaire de Bayle et la lutte philosophique au XVIII siècle, Lyon, Audin, I97I.

Ribard, Dinah, Raconter Vivre Penser, Paris, Vrin, 2003.

Roche, Daniel, Le Siècle des Lumières en province, Académies et académiciens provinciaux, I680-I789, Paris-La Haye, Mouton, I978, vol. I.

Sandrier, Alain, Le Style philosophique du baron d'Holbach : conditions et contraintes du prosélytisme athée en France dans la seconde moitié du XVIII ${ }^{e}$ siècle, Paris, Honoré Champion, 2004.

Viala, Alain, Naissance de lécrivain : sociologie de la littérature à l'âge classique, Paris, Éd. de Minuit, 1985.

Waquet, Françoise, «Pour une éthique de la réception : les "jugements des livres en général" d'Adrien Baillet (I685) ", XVII siècle, avril-juin I988, $\mathrm{n}^{\circ} \mathrm{I59}$, pp. I57-I74.

Waschek, Matthias (dir.), Les «Vies » d'artistes, Paris, Ensa-b, 1996.

Zaborov, Piotr, "Les dictionnaires biographiques russes ", Dix-huitième siècle, Paris, La Découverte, nº 38, 2006. 